\title{
Neoplasias epiteliales del timo: timoma y carcinoma tímico. Caracterización, tratamiento y variables asociadas a supervivencia*
}

\author{
Drs. ROBERTO GONZÁLEZ L. ${ }^{1,2}$, RAIMUNDO SANTOLAYA C., ${ }^{1,2}$, \\ ANDRÉS JADUE T. ${ }^{2}$, RAFAEL PRATS M. ${ }^{1,2}$, GERARDO MORDOJOVICH R. ${ }^{1,2}$, \\ PATRICIO RODRÍGUEZ D. ${ }^{1,2}$ \\ 1 Sección de Cirugía de Tórax, Servicio Médico Quirúrgico Respiratorio, Instituto Nacional del Tórax. \\ 2 Departamento de Cirugía, Campus Oriente, Universidad de Chile. \\ Santiago, Chile.
}

Abstract

\section{Thymic epithelial tumors. Retrospective review of 54 patients}

Background: Thymic epithelial tumors are uncommon and can be associated with myasthenia gravis. Aim: To describe variables associated with survival and treatment of thymic epithelial tumors. Material and Methods: Retrospective review of surgical databases of a respiratory diseases hospital, identifying patients operated for a thymic epithelial tumor between 2000 and 2010. Follow up lasted from 12 to 156 months and information was obtained from medical records and death certificates of the Chilean national identification service. Results: Data from 54 patients aged $52.5 \pm 16.4$ years ( 33 women) was retrieved. Forty two patients were symptomatic and 47 were subjected to resective surgery. The pathological diagnosis was thymoma in 46 cases and thymic carcinoma in eight. Fourteen patients had postoperative complications and one died. Mean survival time was $101.8 \pm 10.2$ months. One, three and five years survival was $90.7 \pm 3.9,81.4 \pm 5.7$ and $71.8 \pm 8.2 \%$, respectively. Preoperative performance status of patients, histological type of the tumor and associated myasthenia gravis were predictors of survival. Conclusions: More commonly, thymic epithelial tumors appear in women, their histological type corresponds to thymomas and their resection is feasible

Key words: Thymoma, thymic carcinoma, myasthenia gravis.

\section{Resumen}

Objetivos: Describir características, tratamiento y variables asociadas a supervivencia de neoplasias epiteliales del Timo (NET). Material y Método: Revisión retrospectiva de pacientes con NET. Período: enero de 2000 - agosto de 2010. Se describen características, tratamiento, morbilidad, mortalidad y supervivencia global. Se comparó supervivencias según variables seleccionadas. Se utilizó programa SPSS 15.0. Se consideró significativo $\mathrm{p}<0,05$. Resultados: 54 pacientes, 33 mujeres, edad promedio 52,5 años. Sintomáticos 42 pacientes. Se realizó cirugía resectiva en 47 . Histología: 46 Timomas y 8 Carcinomas Tímicos. Complica-

*Recibido el 23 de enero de 2012 y aceptado para publicación el 28 de mayo de 2012.

Los autores no refieren conflictos de interés ni apoyo financiero.

Correspondencia: Dr. Roberto González L.

José Manuel Infante 717, Santiago, Chile. Fax: 056-02-5754997.

rgonzalezlagos@udec.c 
ciones en 14 y 1 falleció. Rango seguimiento: 12-156 meses. Tiempo promedio supervivencia 101,8 $\pm 10,2$ meses. Supervivencia global a 1,3 y 5 años: $90,7 \pm 3,9 \%, 81,4 \pm 5,7 \%$ y $71,8 \pm 8,2 \%$ respectivamente. Se encontraron variables asociadas a supervivencia. Conclusiones: Las NET son más frecuentes en mujeres, la mayoría son sintomáticos e histológicamente son timoma. La cirugía es resectiva en la mayoría. Se identifican variables asociadas a supervivencia.

Palabras clave: Timoma, carcinoma tímico, neoplasias tímicas, timo, cirugía torácica, pronóstico.

\section{Introducción}

Las neoplasias epiteliales del timo (NET) son infrecuentes y constituyen las neoplasias malignas más habituales del timo. El timoma (TM), el Carcinoma Tímico (CT) y el Carcinoide Tímico, si bien comparten un origen anatomopatológico epitelial común, poseen características que los distinguen ${ }^{1-4}$.

El TM no presenta atipía evidente del componente epitelial, mantiene las características histológicas del timo y puede estar extensamente infiltrado por linfocitos no neoplásicos. El CT tiene atipía celular, no mantiene las características específicas del timo y equivale aproximadamente al $5 \%$ de las NET. El Carcinoide Tímico es muy infrecuente, se considera un carcinoma neuroendocrino bien diferenciado o carcinoide atípico, está formado por células cuboideas y en su citoplasma se reconocen gránulos neurosecretorios ${ }^{1-6}$.

Los TM y los CT en conjunto constituyen del 0,06 al $1,5 \%$ de todos los tumores, son más frecuentes entre los 40 y 60 años, su etiología se desconoce y aproximadamente el $30 \%$ son asintomáticos al momento del diagnóstico ${ }^{1,3,7-13}$. Existen múltiples clasificaciones o estadificaciones para las NET y su aplicación clínica aún es motivo de discusión 1,3,12,14-21 (Anexo 1).

La cirugía es el tratamiento de elección para la mayoría de los casos y se han descrito variables asociadas a supervivencia ${ }^{1,3,7,8,11,12}$.

Los objetivos de nuestra comunicación son describir las características, tratamiento y variables asociados a supervivencia de NET.

\section{Material y Métodos}

Se realizó una revisión retrospectiva de las bases de datos de cirugía, de protocolos operatorios, de registro de pabellón y de anatomía patológica del Instituto Nacional del Tórax.

El período comprendió desde enero de 2000 hasta agosto de 2010.

Se incluyeron todos los pacientes con NET en que se realizó algún procedimiento quirúrgico y que se certificó con estudio anatomopatológico.

La información se obtuvo desde: fichas clínicas, controles médicos y del Servicio de Registro Civil e Identificación.
Se describen: género, edad, Performance Status, asociación con Miastenia Gravis (MG), síntomas y signos, tratamiento, hallazgos operatorios, tipo histológico, clasificación o estadificación, morbilidad, mortalidad, seguimiento y supervivencia global.

De las clasificaciones o estadificaciones publicadas, seleccionamos las de Masaoka ${ }^{14}$, WHO (World Health Organization) $^{15}$, TNM (1991) ${ }^{16}$, GETT (Gruope d'Etudes des tumeurs Thymiques) ${ }^{17}$ y Bergh ${ }^{18}$. Todos los pacientes fueron clasificados según los hallazgos operatorios, histología y estudio de imágenes (las subetapas en TNM (1991) y GETT fueron agrupadas).

Se comparó supervivencia según 12 variables seleccionadas y según clasificaciones o estadificaciones elegidas. Se utilizaron las pruebas estadísticas de Kaplan-Meier, log-rank y Breslow. Se utilizó programa SPSS 15.0. Se consideró significativo $\mathrm{p}<0,05$.

\section{Resultados}

Durante el período estudiado, en el Instituto Nacional del Tórax se realizaron procedimientos quirúrgicos en 54 pacientes con NET certificadas con estudio anatomopatológico.

La mayoría de los 54 pacientes con NET fueron de género femenino (relación de 1,6/1) y el promedio de edad fue de $52,5 \pm 16,4$ años, con un rango entre los 17 a 86 (Tabla 1).

Al momento del procedimiento quirúrgico el Performance Status fue 0 ó 1 en 43 pacientes, 2 en 10 pacientes y 3 en un paciente.

Tabla 1. Distribución según género y edad de pacientes con NET

\begin{tabular}{|ll|}
\hline Total NET & $\mathbf{5 4}$ \\
Género & \\
Femenino & 33 \\
Masculino & 21 \\
Relación F / M & $1,6 / 1$ \\
Edad (años) & \\
Promedio & $52,5 \pm 16,4$ \\
Mediana & 54,0 \\
Rango & $17-86$ \\
\hline
\end{tabular}


En 17 pacientes la NET se asoció a MG, de estos, 11 eran de género femenino. El tiempo de evolución de la MG tuvo una mediana de 6 meses (rango 1-36). En 16 de los pacientes con MG el tipo histológico de la NET fue TM y la etapa clínica más frecuente era la IIB (según la clasificación de Osserman y Genkins ${ }^{22}$ para la MG) (Tabla 2).

Estaban sintomáticos 42 pacientes. Los síntomas y signos más frecuentes fueron los asociados a MG, dolor torácico, disnea, baja de peso y tos. En 2 pacientes se presentaron síndromes paraneoplásicos distintos a la MG (un síndrome de Cushing y un síndrome nefrótico) (Tabla 3).

El tipo histológico de la NET fue TM en 46 casos y CT en 8 casos. No encontramos casos de Carcinoide Tímico. En 47 pacientes se realizó cirugía resectiva y en 7 el procedimiento quirúrgico fue no resectivo (diagnóstico). La mayoría se abordó por esternotomía y en 38 pacientes se logró resección completa (R0) (Tabla 4).

En 27 de los 54 pacientes se realizaron tratamientos complementarios o distintos a la cirugía. En 20 de los 47 pacientes con cirugía resectiva, se asociaron otros tratamientos (Tablas 5 y 6).

En 15 pacientes existía invasión de estructuras mediastínicas y 8 pacientes tenían 2 o más estructuras invadidas (Tabla 7).

Presentaron complicaciones postoperatorias 14 pacientes, la más frecuente fue la neumonía. Se reoperaron 5 pacientes: 2 por dehiscencia esternal, 2 por hemotórax $\mathrm{y}$ uno por mediastinitis. Una paciente falleció a los 23 días del postoperatorio, corresponde a una mujer de 74 años con TM que evolucionó con dehiscencia esternal y neumonía (Tabla 8).

La mayoría de los pacientes fueron estadificados según Masaoka en etapa I y clasificados según WHO en B3 (Tabla 9).

El seguimiento fue en promedio 46,07 $\pm 37,8$ meses (rango: 12-156). En este período fueron diagnosticadas 3 recidivas en nuestro hospital. La supervivencia global a 1,3 y 5 años fue: $90,7 \pm 3,9 \%$, $81,4 \pm 5,7 \%$ y $71,8 \pm 8,2 \%$ respectivamente (Figura 1).

De las variables seleccionadas para evaluar supervivencia presentaron significación estadística en el análisis el Per-
Tabla 2. Asociación de NET con Miastenia Gravis

\begin{tabular}{|lc|}
\hline Total NET & $\mathbf{5 4}$ \\
Miastenia Gravis & \\
$\quad$ No & 37 \\
$\quad$ Sí & 17 \\
En pacientes con Miastenia Gravis & 17 \\
Tiempo de evolución de Miastenia Gravis (meses) & \\
$\quad$ Promedio & $8,2 \pm 7,8$ \\
Mediana & 6,0 \\
Rango & $1-36$ \\
Histología de NET & \\
Timoma & 16 \\
Carcinoma tímico & 1 \\
Género & \\
Femenino & 11 \\
Masculino & 6 \\
Clasificación clínica de Miastenia Gravis & \\
I & 3 \\
IIA & 3 \\
IIB & 6 \\
III & 5 \\
\hline
\end{tabular}

Tabla 3. Síntomas y signos en pacientes con NET

\begin{tabular}{|lc|}
\hline Síntomas y signos (en 42 pacientes) & \\
Derivados de la Miastenia Gravis (MG) & 17 \\
Dolor torácico & 15 \\
Disnea & 8 \\
Baja de peso & 6 \\
Tos & 6 \\
Síndrome vena cava superior & 3 \\
Síndromes paraneoplásicos (distintos a MG) & 2 \\
\hline
\end{tabular}

Tabla 4. Tipo histológico, tipo de cirugía, vía de abordaje y grado de resección en pacientes con NET

\begin{tabular}{|lrrrrrc|}
\hline & N & R0 & R1 & R2 & TM & CT \\
Neoplasias epiteliales del timo & $\mathbf{5 4}$ & & & & $\mathbf{4 6}$ & $\mathbf{8}$ \\
Cirugía resectiva & & & & & & \\
Vía abordaje & & & & & & \\
Esternotomía & 33 & 26 & 3 & 4 & 25 & 8 \\
VATS & 6 & 6 & 0 & 0 & 6 & 0 \\
Toracotomía & 4 & 2 & 1 & 1 & 3 & 0 \\
Conversión VATS a esternotomía & 2 & 2 & 0 & 0 & 2 & 0 \\
Conversión VATS a toracotomía & 1 & 1 & 0 & 0 & 1 & 0 \\
Toracotomía bilateral (Clamshell) & 1 & 1 & 0 & 0 & 1 & 0 \\
Total & 47 & 38 & 4 & 5 & 39 & 8 \\
Cirugía no resectiva & & & & & & \\
Vía abordaje & & & & & & \\
Biopsia Trucut & 3 & & & & 3 & 0 \\
Mediastinostomía & 2 & & & & 2 & 0 \\
VATS & 1 & & & & 1 & 0 \\
Esternotomía exploradora & 1 & & & & 1 & 0 \\
Total & 7 & & & & 7 & 0 \\
\hline
\end{tabular}

VATS: cirugía video-asistida; R0: resección tumoral completa, R1: resección con tumor residual microscópico, R2: resección con tumor residual macroscópico; TM: Timoma, CT: Carcinoma tímico. 
Tabla 5. Tratamientos en pacientes con NET

\begin{tabular}{|lrrcrr|}
\hline $\begin{array}{l}\text { Tratamientos en } \\
\text { pacientes con NET }\end{array}$ & N & $\begin{array}{c}\text { RTx } \\
\text { Cirugía resectiva }\end{array}$ & $\begin{array}{c}\text { RTx }+ \\
\text { QTx }\end{array}$ & TM & CT \\
R0 & 37 & 16 & 4 & 39 & 8 \\
R1 & 4 & 4 & 0 & 2 & 2 \\
R2 & 5 & 4 & 1 & 2 & 3 \\
Cirugía no resectiva & 7 & 6 & 1 & 7 & 0 \\
Total & 54 & 22 & 5 & 46 & 8 \\
\hline
\end{tabular}

R0: resección tumoral completa, R1: resección con tumor residual microscópico, R2: resección con tumor residual macroscópico; RTx: radioterapia; QTx: quimioterapia; TM: Timoma, CT: carcinoma tímico.

Tabla 7. Invasión local de estructuras mediastínicas en pacientes con NET

\begin{tabular}{|lc|}
\hline Pacientes con Invasión local & 15 \\
\hline Pulmón & 8 \\
\hline Pleura & 8 \\
\hline Grandes Vasos Venosos (VCS, TVBCI) & 6 \\
\hline Pericardio & 5 \\
Grandes Vasos Arteriales (Ao, AP) & 4 \\
\hline $\begin{array}{l}\text { Pacientes con invasión de 2 o más estructuras } \\
\text { mediastínicas }\end{array}$ & 8 \\
\hline
\end{tabular}

VCS: Vena Cava Superior, TVBCI: Tronco Venoso Braquiocefálico Izquierdo, Ao: Aorta, AP: Arteria Pulmonar.

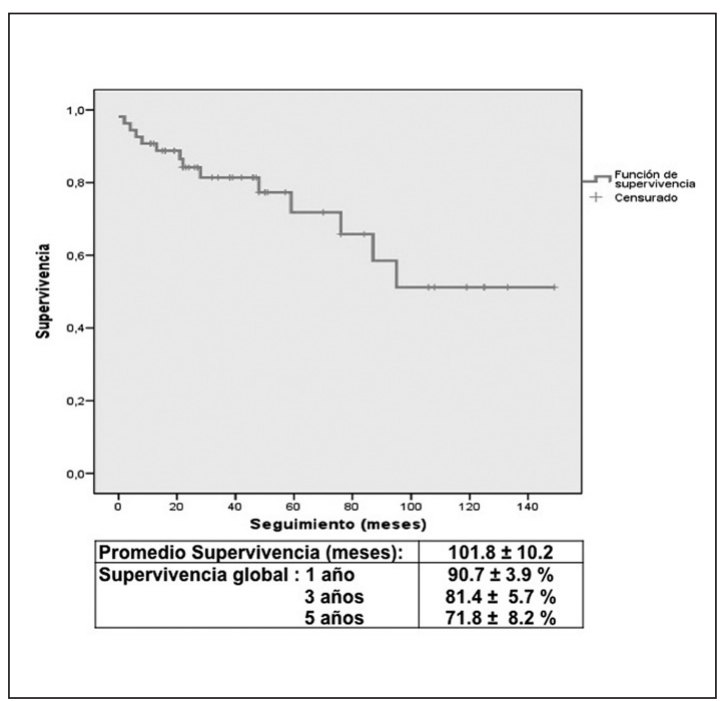

Figura 1. Gráfico de supervivencia global (KaplanMeier) en pacientes con NET.
Tabla 6. Tratamientos en pacientes con NET según etapa de Masaoka

\begin{tabular}{|c|c|c|c|c|}
\hline \multirow[t]{2}{*}{ Masaoka } & \multirow{2}{*}{$\begin{array}{c}n \\
54\end{array}$} & \multicolumn{3}{|c|}{ Tratamiento } \\
\hline & & Cirugía & $\begin{array}{c}\text { Cirugía + } \\
\text { RTx y/o QTx }\end{array}$ & RTx y/o QTx \\
\hline I & 17 & 17 & 0 & 0 \\
\hline II & 14 & 6 & 8 & 0 \\
\hline III & 15 & 4 & 9 & 2 \\
\hline IV & 8 & 0 & 3 & 5 \\
\hline IVA & 5 & 0 & 1 & 4 \\
\hline IVB & 3 & 0 & 2 & 1 \\
\hline
\end{tabular}

RTx: radioterapia; QTx: quimioterapia.

Tabla 8. Complicaciones, reoperaciones y mortalidad en pacientes con NET

\begin{tabular}{|ll|}
\hline Complicaciones & \\
Neumonía & 4 \\
Hemotórax & 2 \\
Dehiscencia esternal & 2 \\
Infección herida operatoria & 2 \\
Crisis Miasténica (Ventilación Mecánica) & 2 \\
Mediastinitis & 1 \\
Quilotórax & 1 \\
Parálisis diafragmática & 1 \\
Taponamiento cardíaco & 1 \\
Disfonía postoperatoria & 1 \\
Reoperaciones & $\mathbf{5}$ \\
Dehiscencia esternal & 2 \\
Hemotórax & 2 \\
Mediastinitis & 1 \\
Mortalidad & 1 \\
\hline
\end{tabular}

Tabla 9. NET según estadificación o clasificación de Masaoka versus WHO

\begin{tabular}{|lccccccc|}
\hline Masaoka & \multicolumn{9}{c}{ WHO } & & Total \\
& A & AB & B1 & B2 & B3 & C & \\
I & 2 & 3 & 7 & 1 & 4 & 0 & 17 \\
II & 1 & 3 & 2 & 2 & 4 & 2 & 14 \\
III & 0 & 2 & 2 & 4 & 3 & 4 & 15 \\
IVA & 0 & 0 & 1 & 3 & 0 & 1 & 5 \\
IVB & 0 & 0 & 0 & 0 & 2 & 1 & 3 \\
Total & 3 & 8 & 12 & 10 & 13 & 8 & 54 \\
\hline
\end{tabular}


formance Status, el tipo histológico, la asociación con MG y en los pacientes resecados la invasión de órganos vecinos y el grado de resección (Tabla $10)$.

De las clasificaciones y estadificaciones selec- cionadas, se correlacionaron adecuadamente con la supervivencia la de Masaoka, TNM (1991) y Bergh (Tabla 11). Para los pacientes con TM (excluidos los CT) la clasificación de Masaoka se correlacionó adecuadamente con la supervivencia (Tabla 12).

Tabla 10. Variables seleccionadas como factores asociados a supervivencia en pacientes con NET

\begin{tabular}{|c|c|c|c|c|c|c|}
\hline \multirow[b]{2}{*}{ Variables } & \multicolumn{5}{|c|}{ Supervivencia } & \multirow[b]{2}{*}{$\mathbf{p}$} \\
\hline & $\mathbf{n}$ & $\begin{array}{c}1 \text { año } \\
\%\end{array}$ & $\begin{array}{c}3 \text { años } \\
\%\end{array}$ & $\begin{array}{c}5 \text { años } \\
\%\end{array}$ & $\begin{array}{l}\text { Promedio } \\
\text { Meses }\end{array}$ & \\
\hline \multicolumn{7}{|l|}{ Género } \\
\hline Femenino & 33 & $95,2 \pm 4,6$ & $95,2 \pm 4,6$ & $76,2 \pm 17,4$ & $96,1 \pm 12,4$ & NS \\
\hline Masculino & 21 & $87,9 \pm 5,7$ & $74,4 \pm 7,9$ & $68,7 \pm 9,1$ & $103,0 \pm 14,0$ & \\
\hline \multicolumn{7}{|l|}{ Edad } \\
\hline 65 años o más & 13 & $92,3 \pm 7,4$ & $72,5 \pm 12,6$ & $72,5 \pm 12,6$ & $97,1 \pm 14,1$ & NS \\
\hline Menos 65 años & 41 & $92,2 \pm 4,6$ & $83,9 \pm 61$ & $73,1 \pm 9,0$ & $102,4 \pm 10,9$ & \\
\hline \multicolumn{7}{|l|}{ Performance status } \\
\hline 1 o menos & 43 & $95,3 \pm 3,2$ & $86,1 \pm 5,9$ & $74,8 \pm 9,1$ & $106,2 \pm 10,7$ & 0,023 \\
\hline 2 o más & 11 & $72,7 \pm 13,4$ & $62,3 \pm 15,0$ & $62,3 \pm 15,0$ & $55,0 \pm 11,5$ & \\
\hline \multicolumn{7}{|l|}{ Histología } \\
\hline Timoma & 46 & $95,7 \pm 3,0$ & $84,7 \pm 5,9$ & $72,1 \pm 5,7$ & $106,0 \pm 11,2$ & 0,036 \\
\hline Carcinoma Tímico & 8 & $62,5 \pm 17,1$ & $62,5 \pm 17,1$ & $62,5 \pm 17,1$ & $68,0 \pm 19,3$ & \\
\hline \multicolumn{7}{|l|}{ Miastenia Gravis } \\
\hline Con Miastenia Gravis & 17 & 100 & 100 & 100 & $\mathrm{NC}$ & 0,0014 \\
\hline Sin Miastenia Gravis & 37 & $86,5 \pm 5,6$ & $72,4 \pm 8,1$ & $58,5 \pm 11,0$ & $83,0 \pm 12,0$ & \\
\hline \multicolumn{7}{|l|}{ Síntomas } \\
\hline Sintomático & 42 & $88,1 \pm 5,0$ & $79,6 \pm 6,5$ & $68,9 \pm 90,1$ & $100,8 \pm 11,1$ & NS \\
\hline Asintomático & 12 & 100 & $87,5 \pm 11,7$ & $87,5 \pm 11,7$ & $90,7 \pm 17,7$ & \\
\hline \multicolumn{7}{|l|}{ Intención cirugía } \\
\hline Curativa (resecados) & 47 & $89,4 \pm 4,5$ & $83,6 \pm 8,5$ & $73,6 \pm 8,4$ & $103,8 \pm 10,4$ & NS \\
\hline Diagnóstica (biopsia) & 7 & $85,7 \pm 13,2$ & $68,6 \pm 18,6$ & $\mathrm{NC}$ & $39,7 \pm 6,1$ & \\
\hline Invasión local en resecados & 47 & & & & & \\
\hline Con invasión local de estructuras vecinas & 15 & $73,3 \pm 11,3$ & $64,2 \pm 12,2$ & $64,2 \pm 12,2$ & $75,2 \pm 15,0$ & 0,019 \\
\hline Sin invasión local de estructuras vecinas & 32 & $96,9 \pm 3,1$ & $93,0 \pm 4,8$ & $75,1 \pm 12,3$ & $115,0 \pm 13,2$ & \\
\hline Grado de resección (R) & 47 & & & & & \\
\hline R0 & 38 & $94,7 \pm 3,6$ & $91,5 \pm 4,7$ & $78,0 \pm 9,8$ & $112,8 \pm 11,4$ & 0,01 \\
\hline R1 o R2 & 9 & $66,7 \pm 15,7$ & $53,3 \pm 17,3$ & $53,3 \pm 17,3$ & $61,6 \pm 18,0$ & \\
\hline Tratamiento en resecados & 47 & & & & & \\
\hline Sólo cirugía & 27 & $96,3 \pm 3,6$ & $91,7 \pm 5,7$ & $83,4 \pm 9,5$ & $120,5 \pm 12,7$ & NS \\
\hline Cirugía + RTx y/o QTx & 20 & $80,0 \pm 8,9$ & $73,3 \pm 10,4$ & $61,1 \pm 14,1$ & $76,1 \pm 11,9$ & \\
\hline \multicolumn{7}{|l|}{ Abordaje (intención tratar) } \\
\hline Abierta (Esterno o Toraco o Clamshell) & 38 & $89,5 \pm 5,0$ & $85,7 \pm 6,0$ & $74,9 \pm 8,9$ & $105,6 \pm 10,8$ & NS \\
\hline Video-asistida (Incluye convertidas) & 9 & $88,9 \pm 10,5$ & $71,1 \pm 18,0$ & $\mathrm{NC}$ & $40,8 \pm 6,6$ & \\
\hline Biopsia & 7 & $85,7 \pm 13,2$ & $68,6 \pm 18,6$ & $\mathrm{NC}$ & $39,7 \pm 6,1$ & \\
\hline \multicolumn{7}{|l|}{ Tratamiento } \\
\hline Sólo Cirugía & 27 & $96,3 \pm 3,6$ & $91,7 \pm 5,7$ & $83.4 \pm 9.5$ & $120,5 \pm 12,7$ & NS \\
\hline Cirugía + RTx y/o QTx & 20 & $80,0 \pm 8,9$ & $73,3 \pm 10,4$ & $61.1 \pm 14.1$ & $76,1 \pm 12,9$ & \\
\hline RTx y/o QTx & 7 & $85,7 \pm 13,2$ & $68,6 \pm 18,6$ & $\mathrm{NC}$ & $39,7 \pm 6,1$ & \\
\hline
\end{tabular}

R0: resección tumoral completa, R1: resección con tumor residual microscópico, R2: resección con tumor residual macroscópico; RTx: radioterapia; QTx: quimioterapia; Esterno: Esternotomía, Toraco: Toracotomía, NC: no calculable, NS: no significativo. 
Tabla 11. Supervivencia según clasificaciones o estadificaciones seleccionadas en pacientes con NET

\begin{tabular}{|c|c|c|c|c|c|c|}
\hline \multirow{2}{*}{$\begin{array}{l}\text { Clasificación o } \\
\text { estadificación }\end{array}$} & \multicolumn{6}{|c|}{ Supervivencia } \\
\hline & $\mathbf{n}$ & $\begin{array}{c}1 \text { año } \\
\%\end{array}$ & $\begin{array}{c}3 \text { años } \\
\%\end{array}$ & $\begin{array}{c}5 \text { años } \\
\%\end{array}$ & $\begin{array}{l}\text { Promedio } \\
\text { Meses }\end{array}$ & p \\
\hline \multicolumn{7}{|l|}{ Masaoka } \\
\hline I & 17 & 100 & 100 & $85,7 \pm 13,2$ & $134,6 \pm 13,4$ & 0,04 \\
\hline II & 14 & $92,6 \pm 6,9$ & $92,6 \pm 6,9$ & $69,6 \pm 20,8$ & $89,9 \pm 14,9$ & \\
\hline III & 15 & $80,0 \pm 10,3$ & $61,7 \pm 14,0$ & $61,7 \pm 14,0$ & $74,3 \pm 15,1$ & \\
\hline IV & 8 & $87,5 \pm 11,7$ & $58,3 \pm 18,6$ & $\mathrm{NC}$ & $34,6 \pm 6,9$ & \\
\hline IVA & 5 & $80,0 \pm 17,9$ & $60,0 \pm 21,9$ & $\mathrm{NC}$ & $35,0 \pm 8,6$ & \\
\hline IVB & 3 & 100 & $50,0 \pm 3,5$ & $\mathrm{NC}$ & $27,5 \pm 10,3$ & \\
\hline \multicolumn{7}{|l|}{ WHO } \\
\hline A & 3 & 100 & 100 & 100 & $\mathrm{NC}$ & NS \\
\hline $\mathrm{AB}$ & 8 & $87,5 \pm 11,7$ & $87,5 \pm 11,7$ & $43,8 \pm 31,5$ & $88,7 \pm 18,8$ & \\
\hline B & 35 & $94,1 \pm 4,0$ & $83,0 \pm 7,0$ & $52,2 \pm 15,5$ & $94,6 \pm 11,2$ & \\
\hline B1 & 12 & $90,0 \pm 8,7$ & $90,0 \pm 8,7$ & $77,9 \pm 14,1$ & $101,0 \pm 11,4$ & \\
\hline B2 & 10 & $90,0 \pm 9,5$ & $78,8 \pm 13,4$ & $78,8 \pm 13,5$ & $107,1 \pm 16,3$ & \\
\hline B3 & 13 & $91,7 \pm 8,0$ & $80,2 \pm 12,8$ & $40,1 \pm 29,1$ & $66,1 \pm 14,3$ & \\
\hline $\mathrm{C}$ & 8 & $62,5 \pm 17,1$ & $62,5 \pm 17,1$ & $31,3 \pm 23,7$ & $68,0 \pm 19,3$ & \\
\hline \multicolumn{7}{|l|}{ TNM (1991) } \\
\hline I & 17 & 100 & $85,7 \pm 13,1$ & $85,7 \pm 13,1$ & $134,6 \pm 13,5$ & 0,035 \\
\hline II & 14 & $92,9 \pm 6,9$ & $92,9 \pm 6,9$ & $69,2 \pm 20,8$ & $89,9 \pm 14,9$ & \\
\hline III & 13 & $76,9 \pm 11,7$ & $53,8 \pm 16,2$ & $53,8 \pm 16,2$ & $66,8 \pm 16,5$ & \\
\hline IV & 10 & $90,0 \pm 9,5$ & $67,5 \pm 15,5$ & $67,5 \pm 15,5$ & $60,9 \pm 11,0$ & \\
\hline \multicolumn{7}{|l|}{ GETT } \\
\hline I & 17 & 100 & $85,7 \pm 13,2$ & $85,7 \pm 13,2$ & $134,6 \pm 13,4$ & NS \\
\hline II & 21 & $95,5 \pm 6,4$ & $84,8 \pm 8,1$ & $72,7 \pm \quad 7,2$ & $91,7 \pm 12,6$ & \\
\hline III & 8 & $75,0 \pm 15,3$ & $40,0 \pm 20,3$ & $40,0 \pm 20,3$ & $51,2 \pm 18,7$ & \\
\hline IV & 8 & $87 \pm 11,7$ & $72,9 \pm 16,5$ & $72,9 \pm 16,5$ & $63,6 \pm 12,4$ & \\
\hline \multicolumn{7}{|l|}{ Bergh } \\
\hline I & 17 & 100 & 100 & $85,7 \pm 13,2$ & $134,6 \pm 13,6$ & 0,017 \\
\hline II & 14 & $92,9 \pm 6,0$ & $92,9 \pm 6,0$ & $69,6 \pm 20,8$ & $89,9 \pm 14,9$ & \\
\hline III & 23 & $82,6 \pm 7,9$ & $60,6 \pm 11,2$ & $60,6 \pm 11,2$ & $73,2 \pm 13,7$ & \\
\hline
\end{tabular}

NC: no calculable, NS: no significativo.

Tabla 12. Supervivencia según etapa Masaoka en pacientes con timoma (excluidos los carcinomas tímicos)

\begin{tabular}{|c|c|c|c|c|}
\hline \multirow[b]{2}{*}{ Etapa } & \multicolumn{4}{|c|}{ Supervivencia timoma } \\
\hline & $\mathbf{n}$ & $\begin{array}{c}5 \text { años } \\
\%\end{array}$ & $\begin{array}{c}\text { Promedio } \\
\text { Meses }\end{array}$ & $\mathbf{p}$ \\
\hline Masaoka & 46 & & & \\
\hline I & 17 & $85,7 \pm 13,2$ & $134,6 \pm 13,4$ & 0,016 \\
\hline II & 12 & $75,0 \pm 21,7$ & $96,3 \pm 14,7$ & \\
\hline III & 11 & $52,6 \pm 18,8$ & $67,9 \pm 19,1$ & \\
\hline IV & 6 & $\mathrm{NC}$ & $39,0 \pm 6,4$ & \\
\hline IVA & 4 & $\mathrm{NC}$ & $42,8 \pm 6,8$ & \\
\hline IVB & 2 & $\mathrm{NC}$ & $27,5 \pm 10,6$ & \\
\hline
\end{tabular}

NC: no calculable.

\section{Discusión}

Las NET son infrecuentes, en Estados Unidos tienen una incidencia estimada de 0,15 por 100.00 personas/año y equivalen aproximadamente al $45 \%$ a $50 \%$ de los tumores de mediastino anterior. Los TM son típicamente de crecimiento lento, producen invasión local y las metástasis frecuentemente son limitadas a pleura, pericardio y diafragma. Las metástasis linfáticas y extratorácicas son infrecuentes ${ }^{1,3,7,8,11-13}$.

Las NET son más frecuentes en el género femenino y la mayoría se presentan entre los 40 y 60 años. En nuestra serie la edad promedio fue 52,5 años y fueron más frecuentes en el género femenino similar a lo comunicado. 
Los síntomas y signos de las NET pueden estar ausentes aproximadamente en el $30 \%$ de los casos. En nuestra serie 12 pacientes estaban asintomáticos al momento del diagnóstico siendo la NET un hallazgo. Los síntomas y signos son inespecíficos como: tos, dolor y disnea; además se pueden presentar síntomas y signos derivados de la compresión de estructuras como síndrome de vena cava superior y con síndromes paraneoplásicos o autoinmunes como: Miastenia Gravis (MG), Artritis Reumatoidea, Lupus Eritematoso, Tiroiditis y síndrome de Sjögren ${ }^{1,3,7-13}$. En nuestra serie los síntomas y signos se presentaron en 42 pacientes y los más frecuentes fueron los derivados de la MG, sólo 2 casos presentaron síndromes paraneoplásicos distintos a la MG.

En los pacientes con NET, la MG está presente entre el $24 \%$ y $47 \%$ de los casos. Por el contrario, en los paciente con MG, aproximadamente el $10 \%$ a $15 \%$ tiene una NET. Múltiples comunicaciones han demostrado que los pacientes con TM y MG tienen mejor pronóstico que los con TM y sin MG, una posible explicación a esto sería el diagnóstico precoz debido a los síntomas de la $\mathrm{MG}^{1,3,11,12,23,24}$. En nuestra serie aproximadamente un tercio de los pacientes presentó MG y esta se asoció significativamente a mejor supervivencia.

Los TM tienden a infiltrar estructuras vecinas y a presentar recurrencia local por sobre las metástasis a distancia. Los CT (también llamados TM tipo C) son más invasivos que los TM, dan más recurrencias y tienen menor supervivencia ${ }^{3,5,7,20,25-27}$. En 15 de nuestros pacientes, existía infiltración de estructuras vecinas y 8 tenían más de una estructura infiltrada, lo que también se asoció significativamente a la supervivencia.

El tratamiento de las NET depende de la etapa evolutiva y se basa en cirugía, radioterapia y quimioterapia. La cirugía es el pilar del tratamiento. La radioterapia y quimioterapia pueden ser utilizadas como tratamiento de inducción, adyuvante o paliativo, sin embargo, la terapia óptima aún es motivo de discusión ${ }^{1,3,7,11,12,24,28,29}$.

No está claro si la radioterapia y la quimioterapia asociada a cirugía mejoran la supervivencia. La radioterapia no es recomendada en etapa I de Masaoka completamente resecada. Para etapa II y III, la radioterapia asociada es controversial. En las NET no completamente resecables o resecadas, la radioterapia ha demostrado beneficios. Para etapa IV o III irresecables, la terapia multimodal (que incluye radioterapia, quimioterapia y eventualmente la cirugía) debe ser considerada ${ }^{1,3,7,11,12,29-31}$. En nuestra serie, de los 47 pacientes resecados, 20 recibieron tratamientos distintos a la cirugía, los 7 pacientes con cirugía no resectiva fueron tratados con radioterapia y/o quimioterapia.
La recurrencia descrita en NET completamente resecados (R0) es de $6,4 \%$ a $8,6 \%$; en tumores resecados con tumor residual microscópico (R1) y en tumores resecados con tumor residual macroscópico (R2) la recurrencia alcanza hasta el $35 \%$ a $50 \%$, en estos pacientes la radioterapia (sobre $50 \mathrm{~Gy}$ ) asociada a cirugía deben ser consideradas ${ }^{1,7,12,24,29}$. Para pacientes con recurrencia local, la reducción de masa tumoral permitiría una supervivencia de $30 \%$ a $50 \%$ a 5 años ${ }^{3,7,24,32}$. Blumberg y cols ${ }^{33}$, comunican que el $68 \%$ de las recurrencias ocurren localmente, Haniuda y $\operatorname{cols}^{34}$, informan que el $92 \%$ de las recurrencias corresponden a diseminación pleural.

En el seguimiento, en nuestro hospital se diagnosticaron 3 recidivas, aunque estas recidivas pueden estar subdiagnosticadas y haber sido tratadas en otros hospitales.

Las vía de abordaje para NET pueden ser cervicotomía, esternotomía, toracotomía, abordajes mínimamente invasivos, cirugía video-asistida (VATS) o robótica. La más utilizada es la esternotomía, sin embargo, no existe suficiente evidencia para recomendar un abordaje. Según diversos autores lo recomendado es que el abordaje permita la resección completa ${ }^{12,24,29,35}$. En nuestra serie la vía de abordaje más utilizada fue la esternotomía.

La mortalidad quirúrgica de las NET es aproximadamente $2,5 \%(0,7 \%-4,9 \%)$ y la morbilidad está dada principalmente por complicaciones respiratorias ${ }^{12,24,29,35}$. En nuestra serie un sólo paciente falleció y la complicación más frecuente fue la neumonía.

Existen múltiples sistemas de clasificación o estadificación para las NET, por lo que determinar cual es el más recomendado aún es motivo de discusión 1,3,12,14-21,26,36. La idea de clasificar los TM fue introducida por Bergh y cols ${ }^{18}$, en 1978, modificada por Wilkins y Castleman y luego por Masaoka y cols $^{14}$, en 1981.

La de Masaoka es la más utilizada y aceptada, se basa en el grado de infiltración de la cápsula tumoral y estructuras vecinas. Se define según criterios clínicos y anatomopatológicos y se considera un excelente predictor independiente de superviven$\mathrm{cia}^{1,4,7,8,14,19,21,24,25,29,37}$.

Sin embargo, de identifican algunos problemas:

- No existe mucha diferencia pronóstica entre etapas I y II.

- Algunas definiciones nos son aplicables completamente a la clínica (incluidas las imágenes) o a la anatomía patológica, en particular en la etapa II.

- No es del todo aplicable a los CT.

- La presencia de tumor residual (R1 o R2) no es considerada, siendo este un conocido factor asociado a supervivencia. 
La clasificación $\mathrm{WHO}^{15}$ de 1999 , es una clasificación histológica. Aunque el tipo histológico se considera un factor independiente de superviven$\mathrm{cia}^{3,36,38-42}$, este sistema de clasificación no permite distinguir los TM biológicamente benignos de los malignos ${ }^{29,42}$. Se considera que el grado de invasión y presencia de metástasis son factores importantes para la supervivencia general ${ }^{7,12,43}$, esto implica el uso de algún criterio de invasión y diseminación que esta clasificación no cuantifica, a diferencia de la de Masaoka. Se ha recomendado revisarla y simplificarla ${ }^{20,27,28}$.

El sistema TNM ha sido propuesto también para las NET y existen varias versiones, la versión de 1991 propuesta por Yamakawa y $\operatorname{cols}^{16}$, ha sido considerada como un excelente factor pronóstico ${ }^{43,44}$. Sin embargo, dadas las características de estas neoplasias que dan pocas metástasis ganglionares y a distancia, esta clasificación ha sido cuestiona$\mathrm{da}$, ya que pocos pacientes tendrían el factor $\mathrm{N} \mathrm{y}$ $\mathrm{M}^{12,21,29,43,44}$.

En Francia, múltiples centros adoptaron la clasificación $\mathrm{GETT}^{17}$, que combina el grado de invasión, de resección y metástasis a distancia. Esta clasificación puede resultar algo confusa y en la práctica clínica es poco utilizada ${ }^{12,29}$.

La clasificación de Bergh y $\operatorname{cols}^{18}$, es simple, fue modificada por Masaoka y al igual que ésta, se basa en el grado de infiltración de la cápsula tumoral y de estructuras vecinas. Tiene los mismos inconvenientes, además que no hace diferencia entre tumores con infiltración y con metástasis.

Utilizando los hallazgos operatorios, procedimientos quirúrgicos, histología y el estudio de imágenes, agrupamos a todos los pacientes según estas 5 clasificaciones o estadificaciones seleccionadas. Encontramos adecuada asociación con la supervivencia en las de Masaoka, TNM (1991) y de Bergh.

La supervivencia comunicada a 5 años según etapa de Masaoka para TM está entre: $85 \%$ a $100 \%$, $70 \%$ a $91 \%, 59 \%$ a $75 \%, 34 \%$ a $71 \%$ y $0 \%$ a $53 \%$ para etapas I, II, III, IVA y IVB respectivamente $\mathrm{e}^{1,3,7,7,11,12,14,21,24}$.

Para las NET se han descrito variables asociadas a mejor supervivencia como: etapa, grado de resección, infiltración de estructuras vecinas, metástasis linfáticas, metástasis hematógenas y asociación a MG entre otras ${ }^{1,7,11,12,23-26,35,43}$.

Kondo y $\mathrm{col}^{7}$, en una serie multicéntrica de 1.320 pacientes, señala que los mejores factores pronósticos para TM son la etapa de Masaoka y la resección completa y para CT la resección completa y las metástasis linfáticas.
La resección completa (R0) está asociada en forma significativa a mejor supervivencia a 5 años, esto es aplicable en etapa Masaoka I, II y III, aun cuando exista infiltración de estructuras vecinas $1,7,12,24,29$. En etapa III la resección extendida (pleurectomía, pericardiectomía, resección pulmonar en cuña, parcial de vena cava y de tronco venoso innominado o braquiocefálico izquierdo) es recomendada ${ }^{12,24,27,29,45}$.

En la serie comunicada por Kondo y $\mathrm{col}^{43}$, las metástasis linfáticas en NET están presentes en el $1,8 \%$ de los TM, en el $27 \%$ de los CT y en el $28 \%$ de los Carcinoides Tímicos. La supervivencia para N0, N1 y N2 de los TM fue $96 \%, 62 \%$ y $20 \%$, para N0, N1, N2 y N3 de los CT fue $56 \%, 42 \%, 29 \%$ y $19 \%$ respectivamente. Cuando existe infiltración de órganos vecinos la diseminación linfática es significativamente mayor y la linfadenectomía es recomendada ${ }^{43}$.

Las metástasis hematógenas en TM están presentes entre el $0 \%$ a $10 \%$ (promedio 5,5\%). Los lugares más frecuentes de metástasis son el pulmón, huesos e hígado. En TM la supervivencia para M0 y M1 es $95 \%$ y $57 \%$, y para CT es $51 \%$ y $35 \%$ respectivamente ${ }^{43}$.

Aunque las metástasis linfáticas y a distancia son factores pronósticos, dadas las características de las NET, estas son poco frecuentes, en la literatura sólo un $2 \%$ de los pacientes tienen metástasis linfáticas y un $1 \%$ metástasis a distancia ${ }^{1,12,43}$.

De las variables seleccionadas en nuestra serie de NET, se asociaron a supervivencia: Performance Status, tipo histológico, asociación a MG, resección completa y la invasión local en resecados.

Un aspecto relevante en el enfrentamiento de las NET, son los significativos avances en biología y patología molecular que recientemente han identificado características moleculares de ellas ${ }^{6,46,47}$. Una caracterización molecular de las NET podría ofrecer estrategias para optimizar el diagnóstico, tratamiento y pronóstico.

A modo de conclusiones de nuestra serie, podemos señalar que las NET son más frecuentes en el género femenino, aproximadamente un tercio se asocia a MG, la mayoría son sintomáticos e histológicamente corresponden a timoma. La cirugía es resectiva en la mayoría de los casos, además se identifican variables asociadas a supervivencia.

\section{Agradecimientos}

A nuestras secretarias Sra. Lidia León F. y Sra. Laura Cortés A., por su constante y desinteresado apoyo en la preparación de esta comunicación. 


\section{Anexo 1. Clasificaciones o estadificaciones seleccionadas de NET}

\section{Masaoka $^{14}$}

- I: Macroscópicamente completamente encapsulado y microscópicamente sin invasión capsular

- II: Invasión macroscópica hacia el tejido adiposo circundante o pleura mediastínicas; invasión microscópica hacia la cápsula

- III: Invasión macroscópica hacia los órganos vecinos (pericardio, pulmones y grandes vasos)

- IVA: Diseminación pleural o pericárdica

- IVB: Metástasis linfáticas o hematógenas

\section{WHO (World Health Organization) ${ }^{15}$ :}

- A: Se compone de células epiteliales tímicas neoplásicas con forma de huso u ovales, carecen de atipía nuclear o están acompañadas de linfocitos no neoplásicos

- AB: Los focos que tienen características de timoma tipo A se mezclan con focos ricos en linfocitos no neoplásicos

- B1: Se asemeja al timo funcional normal porque contiene un gran número de células cuya apariencia es casi indistinguible de las células de la corteza tímica normal, con áreas que se asemejan a la médula tímica

- B2: el componente epitelial neoplásico aparece en forma de células rollizas dispersas con núcleos vesiculares y nucléolos diferenciados entre una población extremadamente numerosa de linfocitos no neoplásicos

- B3: se compone predominantemente de células epiteliales de forma redonda o poligonal, y no presenta atipía o presenta atipía leve

- C: presenta una clara atipia citológica y un conjunto de características citológicas no específicas del timo, sino más bien similares a aquellas características citológicas que se observan en los carcinomas de otros órganos

\section{TNM (1991) ${ }^{16}$ :}

- T1: Macroscópicamente completamente encapsulado y microscópicamente sin invasión capsular

- T2: Invasión macroscópica hacia el tejido adiposo circundante o pleura mediastínicas o invasión microscópica hacia la cápsula

- T3: Invasión macroscópica hacia los órganos vecinos (pericardio, pulmones y grandes vasos)

- T4: Diseminación pleural o pericárdica

- N0: Sin metástasis en linfonodos

- N1: Metástasis en linfonodos del mediastino anterior

- N2: Metástasis en linfonodos intratorácicos excepto en linfonodos del mediastino anterior

N3: Metástasis en linfonodos extratorácicos

- M0: Sin metástasis hematógenas

M1: Metástasis Hematógenas

- Etapa I: T1-T2-T3

- Etapa II: T4-N0

- Etapa III: N1-N2

- Etapa IVA: N3-M0

- Etapa IVB: M1

\section{GETT (Gruope d'Etudes des tumeurs Thymiques) ${ }^{17}$ :}

- IA: Encapsulado, resección completa

- IB: Resección macroscópica completa, pero sospecha de adhesiones mediastinales o potencial invasión capsular

- II: Tumor invasivo, resección completa

- IIIA: Tumor invasivo, resección subtotal

- IIIB: Tumor invasivo, sólo se realiza biopsia

- IVA: Metástasis supraclaviculares o pleurales

- IVB: Metástasis a distancia

\section{Bergh $^{18}$ :}

- I: Macroscópicamente completamente encapsulado y microscópicamente sin invasión capsular

- II: Invasión macroscópica hacia el tejido adiposo circundante o pleura mediastínicas; invasión microscópica hacia la cápsula

- III: Invasión macroscópica hacia los órganos vecinos (pericardio, pulmones y grandes vasos), metástasis intratorácicas o ambos 


\section{Referencias}

1. Baas P, Rhodius R. Thymoma update 2011. Eur J Cancer 2011;47 Suppl 3:S315-6.

2. Marchevsky AM, McKenna RJ Jr, Gupta R. Thymic epithelial neoplasms: a review of current concepts using an evidence-based pathology approach. Hematol Oncol Clin North Am. 2008;22:543-62.

3. Kurup A Loehrer PJ St. Thymoma and thymic carcinoma: therapeutic approaches. Clin Lung Cancer 2004;6:28-32.

4. Weydert JA, De Young BR, Leslie KO; Association of Directors of Anatomic and Surgical Pathology. Recommendations for the reporting of surgically resected thymic epithelial tumors. Am J Clin Pathol. 2009;132:10-5.

5. Moran CA, Suster S. Thymic carcinoma: current concepts and histologic features. Hematol Oncol Clin North Am. 2008;22:393-407.

6. Ströbel P, Hohenberger P, Marx A. Thymoma and thymic carcinoma: molecular pathology and targeted therapy. J Thorac Oncol. 2010;5(10 Suppl 4):S286-90.

7. Kondo K, Monden Y. Therapy for thymic epithelial tumors: a clinical study of 1,320 patients from Japan. Ann Thorac Surg. 2003;76:878-84.

8. Sumiyama Y, Yoshida Y. Thymoma. Ann Thorac Cardiovasc Surg. 2004;10:321-3.

9. Sperling B, Marschall J, Kennedy R, Pahwa P, Chibbar R. Thymoma: a review of the clinical and pathological findings in 65 cases. Can J Surg. 2003;46:37-42.

10. Harris K, Elsayegh D, Azab B, Alkaied H, Chalhoub M. Thymoma calcification: is it clinically meaningful? World J Surg Oncol. 2011;9:95.

11. Johnson SB, Eng TY, Giaccone G, Thomas CR Jr. Thymoma: update for the new millennium. Oncologist 2001;6:239-46.

12. Tomaszek S, Wigle DA, Keshavjee S, Fischer S. Thymomas: review of current clinical practice. Ann Thorac Surg. 2009;87:1973-80.

13. Engels EA. Epidemiology of thymoma and associated malignancies. J Thorac Oncol. 2010;5 (10 Suppl 4):S260-5.

14. Masaoka A, Monden Y, Nakahara K, Tanioka T. Follow-up study of thymomas with special reference to their clinical stages. Cancer 1981;48:2485-92.

15. Rosai J. Histological typing of tumours of the thymus. In: WHO International histological classification of tumours, $2^{\text {nd }}$ ed. New York: Springer-Verlag, 1999:5-15.

16. Yamakawa Y, Masaoka A, Hashimoto T, Niwa H, Mizuno T, Fujii Y, et al. A tentative tumor-node-metastasis classification of thymoma. Cancer 1991;68:1984-7.

17. Gamondès JP, Balawi A, Greenland T, Adleine P, Mornex JF, Zhang J, et al. Seventeen years of surgical treatment of thymoma: factors influencing survival. Eur J Cardiothorac Surg. 1991;5:124-31.

18. Bergh NP, Gatzinsky P, Larsson S, Lundin P, Ridell B. Tumors of the thymus and thymic region: I. Cli- nicopathological studies on thymomas. Ann Thorac Surg. 1978;25:91-8.

19. Masaoka A. Staging system of thymoma. J Thorac Oncol. 2010;5(10 Suppl 4):S304-12.

20. Marchevsky AM, Gupta R, McKenna RJ, Wick M, Moran $\mathrm{C}$, Zakowski MF, et al. Evidence-based pathology and the pathologic evaluation of thymomas: the World Health Organization classification can be simplified into only 3 categories other than thymic carcinoma. Cancer 2008;112:2780-8.

21. Asamura H, Nakagawa K, Matsuno Y, Suzuki K, Watanabe S, Tsuchiya R. Thymoma needs a new staging system. Interact Cardiovasc Thorac Surg. 2004;3:163-7.

22. Osserman KE, Genkins G. Studies in myasthenia gravis: review of a twenty-year experience in over 1200 patients. Mt Sinai J Med. 1971;3:497-537.

23. Kondo K, Monden Y. Thymoma and myasthenia gravis: a clinical study of 1,089 patients from Japan. Ann Thorac Surg. 2005;79:219-24.

24. Davenport E, Malthaner RA. The role of surgery in the management of thymoma: a systematic review. Ann Thorac Surg. 2008;86:673-84.

25. Hosaka Y, Tsuchida M, Toyabe S, Umezu H, Eimoto T, Hayashi J. Masaoka stage and histologic grade predict prognosis in patients with thymic carcinoma. Ann Thorac Surg. 2010;89:912-7.

26. Wick MR. Prognostic factors for thymic epithelial neoplasms, with emphasis on tumor staging. Hematol Oncol Clin North Am. 2008;22:527-42.

27. Kim DJ, Yang WI, Choi SS, Kim KD, Chung KY. Prognostic and clinical relevance of the World Health Organization schema for the classification of thymic epithelial tumors: a clinicopathologic study of 108 patients and literature review. Chest 2005;127:755-61.

28. Ruffini E, Van Raemdonck D, Detterbeck F, Rocco G, Thomas P, Venuta F. Management of thymic tumors: a survey of current practice among members of the European Society of Thoracic Surgeons. J Thorac Oncol. 2011;6:614-23.

29. Kondo K. Optimal therapy for thymoma. J Med Invest. 2008;55:17-28.

30. Chang JH, Kim HJ, Wu HG, Kim JH, Kim YT. Postoperative radiotherapy for completely resected stage II or III thymoma. J Thorac Oncol. 2011;6:1282-6.

31. Bott MJ, Wang H, Travis W, Riely GJ, Bains M, Downey R, et al. Management and outcomes of relapse after treatment for thymoma and thymic carcinoma. Ann Thorac Surg. 2011;92:1984-92.

32. Margaritora S, Cesario A, Cusumano G, Lococo F, Porziella V, Meacci E, et al. Single-centre 40-year results of redo operation for recurrent thymomas. Eur J Cardiothorac Surg. 2011;40:894-900.

33. Blumberg D, Port JL, Weksler B, Delgado R, Rosai $\mathrm{J}$, Bains MS, et al. Thymoma: a multivariate analysis of factors predicting survival. Ann Thorac Surg. 1995;60:908-13. 
34. Haniuda M, Morimoto M, Nishimura H, Kobayashi O, Yamanda T, Iida F. Adjuvant radiotherapy after complete resection of thymoma. Ann Thorac Surg. 1992;54:311-5.

35. Demirci S, Turhan K, Ozsan N, Yalman D, Cakan A, Cok G, et al. Prognostic factors for survival in patients with thymic epithelial tumors. Thorac Cardiovasc Surg. 2011;59:153-7.

36. Kim BK, Cho BC, Choi HJ, Sohn JH, Park MS, Chang $\mathrm{J}$, et al. A single institutional experience of surgically resected thymic epithelial tumors over 10 years: clinical outcomes and clinicopathologic features. Oncol Rep. 2008;19:1525-31.

37. Gupta R, Marchevsky AM, McKenna RJ, Wick M, Moran $\mathrm{C}$, Zakowski MF, et al. Evidence-based pathology and the pathologic evaluation of thymomas: transcapsular invasion is not a significant prognostic feature. Arch Pathol Lab Med. 2008;132:926-30.

38. Marchevsky AM, Gupta R, Casadio C, Hiroshima K, Jambhekar NA, Kim DJ, et al. World Health Organization classification of thymomas provides significant prognostic information for selected stage III patients: evidence from an international thymoma study group. Hum Pathol. 2010;41:1413-21.

39. Rena O, Papalia E, Maggi G, Oliaro A, Ruffini E, Filosso P, et al. World Health Organization histologic classification: an independent prognostic factor in resected thymomas. Lung Cancer 2005;50:59-66.

40. Nakagawa K, Asamura H, Matsuno Y, Suzuki K, Kon- do H, Maeshima A, et al. Thymoma: a clinicopathologic study based on the new World Health Organization classification. J Thorac Cardiovasc Surg. 2003;126:1134-40.

41. Park MS, Chung KY, Kim KD, Yang WI, Chung JH, Kim YS, et al. Prognosis of thymic epithelial tumors according to the new World Health Organization histologic classification. Ann Thorac Surg. 2004;78:992-7.

42. Kondo K, Yoshizawa K, Tsuyuguchi M, Kimura S, Sumitomo M, Morita J, et al. WHO histologic classification is a prognostic indicator in thymoma. Ann Thorac Surg. 2004;77:1183-8.

43. Kondo K, Monden Y. Lymphogenous and hematogenous metastasis of thymic epithelial tumors. Ann Thorac Surg. 2003;76:1859-64.

44. Kondo K. Tumor-node metastasis staging system for thymic epithelial tumors. J Thorac Oncol. 2010;5(10 Suppl 4):S352-6.

45. Yang HC, Yoon YS, Kim HK, Choi YS, Kim K, Shim $\mathrm{YM}$, et al. En bloc extended total thymectomy and extrapleural pneumonectomy in Masaoka stage IVA thymomas. J Cardiothorac Surg. 2011;6:28.

46. Chau NG, Kim ES, Wistuba I. The multidisciplinary approach to thymoma: combining molecular and clinical approaches. J Thorac Oncol. 2010;5(10 Suppl 4):S3137.

47. Mimae T, Tsuta K, Takahashi F, Yoshida A, Kondo T, Murakami Y, et al. Steroid receptor expression in thymomas and thymic carcinomas. Cancer 2011;117:4396-405. 